\title{
Technology takes the wheel
}

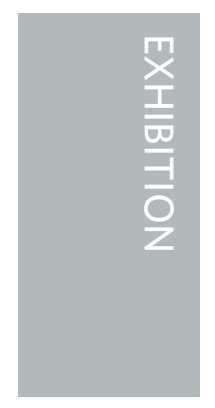

The car of tomorrow will drive itself, using sensors and artificial intelligence to navigate and make decisions. These autonomous vehicles could make roads safer and help reduce emissions. Car companies and research centres around the globe have been developing autonomous technologies for years. But how close are we to letting technology take the wheel?

A new exhibition, Driverless: Who is in Control?, at the Science Museum, London, showcases various autonomous technologies and explores their potential impact on society. It also examines the challenges that exist in their development - the difficulties in deploying fully autonomous vehicles on roads and the limitations of current technology.

The exhibition is divided into three main sections - Land, Air and Water covering the different environments in which driverless technologies can be used. Land includes vehicles that are already in use, but only in controlled environments. The Westfield POD vehicle, for example, has been transporting passengers at Heathrow airport in London since 2011. Its short journeys take place along guideways, but more recently, a newer version of the POD was tested in public spaces in Greenwich, London, and was able to share the road with pedestrians, cyclists and animals. Also on display is the impressive Robocar, a driverless electric racing car that can compete on pre-mapped racetracks and uses an autonomous vehicle computing platform from Nvidia.

The exhibition also features vehicles made for tasks other than transportation. One of these is Tom - a farm monitoring robot developed by the Small Robot Company. Tom is equipped with laser imaging, detection and ranging (lidar) and GPS systems to navigate a pre-mapped field, cameras and sensors to 'see' the crops, and microcontrollers to process information. With Tom's help and an artificial intelligence (AI) interface, two other smart robots can be directed to feed and spray crops.

In Air, remote-controlled drones are shown to solve problems in areas where access by humans is difficult. It highlights, for example, that, during tests in Sweden, drones were able to deliver defibrillators faster than on-road medical services. There is also a video about Manta, an unmanned

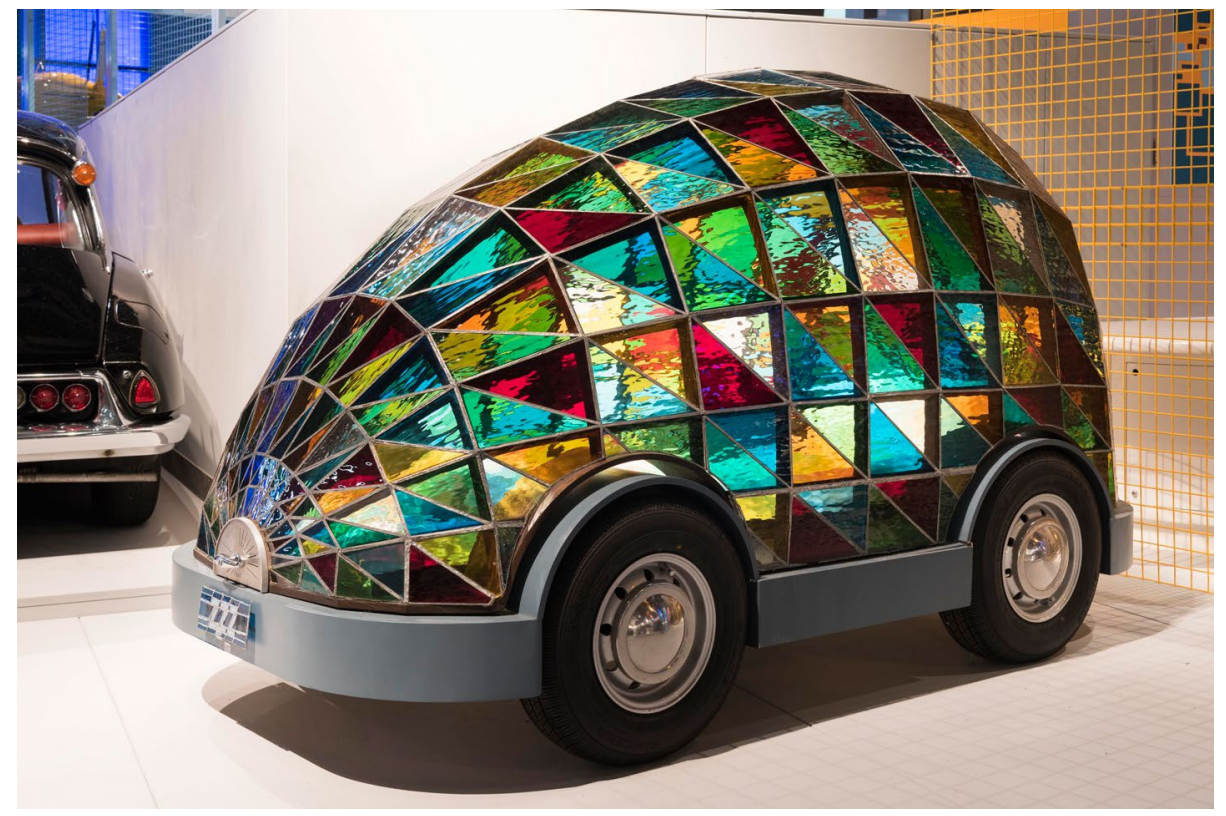

In the future, driverless cars might be safe enough to transport sleeping passengers and to be made of glass. The Stained Glass Driverless Sleeper Car of the Future designed by Dominic Wilcox, on display at Driverless: Who is in Control?, is one such prototype. Credit: (c) Science Museum Group

aerial vehicle with detectors that can identify the location of landmines and help clear them remotely.

Similarly, in Water, vehicles are shown that can explore deep-ocean environments, where humans cannot go. One of these is a scale model of the WAM-V surface vessel used by TeamTao, a finalist in the Shell Ocean Discovery XPrize competition. The vessel has autonomous capabilities and can be used to launch and retrieve a swarm of undersea drones that can map the ocean floor.

Some of the displays explain the clever technology behind the driverless vehicles, such as object-recognition software and systems for navigation like lidar. Visitors are encouraged to test the example software (which was developed by MathWorks) by holding different images in front of a camera, and its capabilities in terms of recognizing and labelling objects are impressive.

But elsewhere in the section, the challenges involved in developing autonomous cars quickly become obvious: an experiment is described in which stickers were put on road signs to confuse a car's image recognition software. The real world, and especially public roads in cities, is full of complex and unpredictable traffic scenarios - pedestrians looking at their phones, children running into traffic - that have to be identified and handled by the car's computer. Training the AI models for all possible situations is simply too big a task.

Letting AI take over from humans at the wheel also creates serious ethical dilemmas. Visitors can try the Moral Machine experiment, a platform developed by researchers at Massachusetts Institute of Technology to gather human perspectives on moral decisions made by machine intelligence. The experiment asks visitors to choose between two scenarios, where the autonomous car kills either the passengers or the pedestrians. The results from the millions of responses the experiment has received show that human moral preferences are complex, with substantial cultural variations. But such insights will definitely be important in determining how machine ethics are programmed.

Driverless: Who is in Control? runs at the Science Museum, London, until October 2020.

Reviewed by Christiana Varnava
Associate Editor, Nature Electronics.
e-mail: christiana.varnava@nature.com

Published online: 15 August 2019 https://doi.org/10.1038/s41928-019-0295-1 